\title{
NUEVOS REGISTROS DE PLANTAS DE LAS MONTAÑAS DEL NORTE DE MEXICO
}

\author{
Richard SpellenberG \\ Department of Biology, New Mexico State University \\ Las Cruces, NM 88003-0001, E.U.A. \\ Toutcha LeBgue \\ Facultad de Zootecnia \\ Universidad Autónoma de Chihuahua \\ Cd. Universitaria, Apdo. Postal F-28 \\ Chihuahua, Chihuahua, México \\ Rafael Corral-Diaz \\ Colegio de Graduados \\ Escuela Superior de Agricultura "Hermanos Escobar" \\ Apdo. Postal 2119 \\ Ciudad Juárez, Chihuahua, México \\ Y \\ JEFFREY BACON \\ Department of Biology, New Mexico State University \\ Las Cruces, NM 88003-0001, E.U.A.
}

\section{RESUMEN}

Se encontraron 20 nuevos registros repartidos en 13 familias para las montañas del norte de México. La mayor parte de estos hallazgos se localizaron en la zona del Parque Nacional "Cascada de Basaseachic", Chihuahua, y son el resultado de los estudios florísticos llevados a cabo por los autores en los últimos siete años.

\section{ABSTRACT}

Twenty new records in 13 families from the mountains of northern Mexico are reported. Most were derived from our floristic studies in the Parque Nacional de la Cascada de Basaseachic, Chihuahua, during the last seven years.

\section{INTRODUCCION}

En los años recientes el acceso a las diferentes áreas de las montañas en el norte de México ha mejorado con la construcción de caminos asociada con las industrias 
agropecuarias, forestales y turísticas. Este desarrollo facilita la exploración botánica. El presente trabajo es el resultado de tales exploraciones y contribuye al conocimiento biogeográfico de esta vasta región.

Asimismo, los autores han llevado a cabo estudios sobre el Parque Nacional "Cascada de Basaseachic", Chihuahua (abreviado en la lista como PNCB), un lugar único por su espectacular belleza y variada vegetación. Los microambientes creados por los precipicios, el rocío de la cascada, y los bosques húmedos en el fondo de la barranca albergan algunas plantas endémicas (Nesom, 1989). La abundancia de microambientes y la localización fisiográfica del Parque son factores que permiten la existencia de especies típicas de regiones ubicadas más hacia el norte y hacia el sur, cuya distribución geográfica se amplía con estos nuevos registros.

El Parque está situado en la vertiente oeste de la Sierra Madre Occidental (108012'30" $\mathrm{W}, 28^{\circ} 11^{\prime} \mathrm{N}$ ), teniendo una variación altitudinal que va desde los $1550 \mathrm{~m}$ en el fondo del cañón hasta $2150 \mathrm{~m}$ en las montañas del área. En su mayor parte, la vegetación se compone de bosque seco de coníferas y Quercus (Rzedowski, 1978) sobre un lecho rocoso de origen ígneo con suelo escaso de color claro. Sin embargo, en el fondo de la barranca y en las pendientes cálidas con exposición suroeste, existe la influencia del bosque tropical caducifolio, el cual se distribuye ampliamente en zonas tropicales más al sur de la cascada. Por ejemplo, en algunos sitios cálidos se encuentra Asclepias ovata, una especie conocida del sur de la Sierra Madre Occidental. En altitudes mayores, especialmente en las pendientes frescas con exposición norte, se localizan plantas que comunmente se distribuyen en regiones más septentrionales, como Amelanchier utahensis y Cardamine breweri. Estas especies, al igual que un buen número de otros registros del Parque, acentúan su singularidad ecológica y la conveniencia de la protección del área. Hay pocos trabajos sobre la flora de la Sierra Madre de Chihuahua y Sonora. A Gentry (1942) se debe una publicación sobre sus estudios de las plantas del Río Mayo, del cual el Río Candameña en Basaseachic es un afluente, pero el mencionado autor no visitó el área cerca de Basaseachic. En la actualidad, el Dr. Paul Martin de la Universidad de Arizona, está incrementando la lista de las plantas de la cuenca del Río Mayo y sus afluentes con muchas colectas nuevas.

En el listado siguiente las siglas "ESAHE" representan al Herbario de la Escuela Superior de Agricultura "Hermanos Escobar", y "CHIH" al Herbario de la Facultad de Zootecnia, Universidad Autónoma de Chihuahua. La información de las colecciones se presenta de acuerdo con las etiquetas que acompañan a los especímenes de herbario respectivos, seguida de comentarios propios a cada registro.

\section{LISTADO DE NUEVOS REGISTROS PARA EL NORTE DE LA SIERRA MADRE OCCIDENTAL, MEXICO}

\section{APIACEAE}

Hydrocotyle pusilla A. Richard. Chihuahua, Mpio. Ocampo, PNCB, in the "box canyon" at the base of the falls, 12 Sep 1986, Spellenberg, Jewell \& Columbus 9295 (ESAHE, MEXU, NMC, UC). La especie se presenta desde el Caribe y México hasta Argentina (Mathias \& Constance, 1976). Constance (com. pers.) indica que UC posee un espécimen proveniente 
Spellenberg, et al.: Nuevos Registros de Plantas de las Montañas del Norte de México

de Jalisco. Este registro extiende la distribución conocida hasta aproximadamente $700 \mathrm{~km}$ al norte. En el Parque crece entre otras plantas herbáceas humedecidas por el rocío de la cascada.

\section{ASCLEPIADACEAE}

Asclepias ovata Mart. \& Gal. Chihuahua, Mpio. Ocampo, PNCB, more or less open, dry Pinus durangensis-Quercus hypoleucoides woods, 14 Sep 1987, Spellenberg \& Jewell 9308 (NMC). Woodson (1954) indica que la especie se localiza desde el centro de México hasta la parte norte de la frontera de Durango y Sinaloa, por lo que esta colección amplía su distribución geográfica en alrededor de $300 \mathrm{~km}$ hacia el norte. Dentro del Parque la especie se encuentra sobre pendientes con exposición este o sur.

\section{ASTERACEAE}

Antennaria marginata E. Greene. Chihuahua, Mpio. Ocampo, 8 mi toward San Juanito from junction of Tomochic-Yepachic road, ca. $16 \mathrm{mi}$ from Basaseachic, on steep $\mathrm{N}$ slope at elev. 8000 ft, 24 Apr 1985, Spellenberg et al. 8064 (ASU, ENCB, MEXU, NMC, NY, TEX, UAC, UC, WOCB). Esta colecta amplía la distribución geográfica de la especie en aproximadamente $250 \mathrm{~km}$ al sursureste, tomando en cuenta lo establecido por Bayer y Stebbins (1987).

\section{BRASSICACEAE}

Cardamine breweri S. Wats. var. breweri. Chihuahua, Mpio. Ocampo, PNCB, in shute-like canyon to $\mathrm{S}$ of falls along trail to base, in spring, with mixed pine and oaks, elev. ca. 1800 m, 26 Apr 1985, Spellenberg et al. 8046 (ESAHE, GH, MEXU, NMC); 9822 (NMC, GH); 17 Mar 1986, Donohue, Martin, Morr \& Lindquist s.n. (ARIZ); at base of the canyon below the falls on a steep $\mathrm{N}$-facing permanently wet slope, on slick wet rock, elev. 1570 m, 11 Nov 1989, Spellenberg, Corral \& Lebgue 10061 (CHIH, CIIDIR, ESAHE, MEXU, MO, NMC, NY, RSA); $5.5 \mathrm{~km}$ W of Huajumar on road to Ocampo, in moist "grotto", 19 Jun 1993, Spellenberg, Brouillet \& Ulaszek 11907 (CIIDIR, IBUG, MEXU, MO, NMC). La especie es común en las montañas del oeste de E.U.A. y el suroeste de Canadá, pero anteriormente no se había encontrado en México. Este registro extiende la distribución conocida hasta aproximadamente $960 \mathrm{~km}$ al sureste de las montañas de San Bernadino en el sur de California. El material de nuestras colectas es muy similar al ilustrado por Hickman (1993, p. 409).

\section{ERIOCAULACEAE}

Eriocaulon bilobatum Morong. Chihuahua, Mpio. Ocampo, PNCB, in canyon along Rio Candameña leading to the falls, 4 Oct 1986, Spellenberg, Soreng, Corral \& Lebgue 8776 (ESAHE, MEXU, NMC, NY, TEX); 25 Sep 1988, Spellenberg \& Boecklen 9752 (ARIZ, MEXU, NMC). Las plantas crecen en algunas pozas a lo largo del Río Basaseachic y brotan cada año. De acuerdo con Moldenke (1937), la especie habita en lugares húmedos en los alrededores de Guadalajara, Jalisco. Posteriormente Moldenke (1975) indica que sólo se 
encuentra en Jalisco. Hace poco la planta se citó para Durango (González Elizondo, González Elizondo y Herrera Arrieta, 1991), pero sin citar una muestra de referencia. Aparentemente nuestras colecciones representan los primeros registros para Chihuahua, ya que no se le menciona para este estado en los trabajos de Lot et al. (1986). La nueva localidad se encuentra aproximadamente a $900 \mathrm{~km}$ al norte de las poblaciones de Jalisco.

\section{FABACEAE}

Astragalus cobrensis A. Gray var. maguirei Kearney. Sonora, Cajon Bonito, creek bottom in area of mesquite, cottonwood, sycamore, elev. ca. $4000 \mathrm{ft}, 2$ May 1974, Todsen 4015 (NMC). La localidad se sitúa en la Sierra de San Luis cerca del extremo noreste del estado de Sonora, en la frontera con Chihuahua y aproximadamente $16 \mathrm{~km}$ al sur de la de Estados Unidos. Barneby (1964) indica que la distribución geográfica del taxon alcanza su extremo meridional a unos $55 \mathrm{~km}$ del límite internacional. Sin embargo, después de la mencionada publicación, se ha encontrado la planta muy cerca de la frontera, en la parte suroeste del estado de Nuevo México. Se cree que esta colecta representa el primer registro para México.

Astragalus daleae Greene. Chihuahua, Mpio. Ocampo, PNCB, along benches of Rio Basaseachic about 100 m above falls, sandy soil, elev. 2000 m, 12 Sep 1987, Spellenberg \& Jewell 9288 (MEXU, NMC, NY). Hallamos solamente una planta en este lugar y nada caminando río más arriba. Esta colecta amplía la distribución geográfica conocida de la especie en aproximadamente $550 \mathrm{~km}$ al norte, tomando en cuenta lo establecido por Barneby (1964).

\section{FAGACEAE}

Quercus gentryi Mull. Chihuahua, Mpio. Guadalupe y Calvo, 43 km SW of Guadalupe y Calvo on road to Galeana, elev. 1480 m, 13 Jul 1986, Spellenberg \& Zimmerman 8554 (CAS, ESAHE, MEXU, NMC), 8559 (CAS, MEXU, NMC). La especie es común sobre las pendientes más secas. El registro es al parecer el primero para el estado de Chihuahua; González Villarreal (1986) comenta que la distribución conocida se extiende desde Sinaloa y Durango hasta Michoacán y Guanajuato.

Quercus subspathulata Trel. Chihuahua, Mpio. Moris, $10 \mathrm{mi}$. E of Moris, $11 \mathrm{mi}$. W of Ocampo, elev. $5000 \mathrm{ft}$ steep $\mathrm{W}$ slope on heavily mineralizad rocky, almost barren soil, 13 Sep 1987, Spellenberg \& Jewell 9388 (BH, CAS, CIIDIR, MEXU, NMC). De acuerdo con González Villarreal (1986), el límite septentrional de la distribución geográfica de la especie se localiza en el norte de Sinaloa, quizás $200 \mathrm{~km}$ al sur del lugar de la colecta mencionada arriba. Se observó que $Q$. subspathulata se asocia con $Q$. viminea Trel., Q. albocincta Trel. y $Q$. chihuahuensis Trel. La especie ha sido recolectada también en Sonora, muy cerca de los límites con Chihuahua, en el Ejido Aserraderos, por P. Martin y colaboradores, 6 Nov 1986 (ARIZ, NMC; fotocopia NMC).

\section{LAMIACEAE}

Salvia summa A. Nels. Mpio. Juarez, Sierra Juarez on W side of Cd. Juarez, ca. $6 \mathrm{~km} \mathrm{~S}$ of US-Mexico border, $106^{\circ} \mathrm{W}, 31^{\circ} 42^{\prime} \mathrm{N}$, elev. ca. $1380 \mathrm{~m}, \mathrm{~N}$-facing limestone cliffs, 
27 Dec 1991, Spellenberg and Bacon 10981 (NMC); in Sierra Juarez in about middle of range, on road to microwave tower, ca. 3 air km SW of Cd. Juarez, elev. $1450 \mathrm{~m}, 7$ May 1993, Spellenberg \& Brouillet 11795 (MEXU, MT, NMC). Correll and Johnston (1970) indican que la especie está restringida al oeste de Texas y sur de Nuevo México. Este es el primer registro para México.

\section{LENTIBULARIACEAE}

Pinguicula oblongiloba DC. Chihuahua, Mpio. Ocampo, PNCB, elev. ca. $2100 \mathrm{~m}$, on mossy, moist, N-facing cliffs beneath Divisadero Overlook, 31 Jul 1989, Spellenberg, Corral, Brunt \& Huenneke 9558 (ESAHE, MEXU, NMC). Anteriormente registrada desde Oaxaca hasta Durango, por lo que la distribución geográfica conocida de esta especie se amplía por lo menos $350 \mathrm{~km}$ hacia el norte. Otra colección en la misma localidad se ha llevado a cabo por Martin et al. s.n. 22 Jun 1986 (ARIZ). La separación de P. macrophylla, P. moranensis y $P$. oblongiloba es difícil y algunos autores como McVaugh y Mickel las consideran como una sola especie; de ser así, el nombre apropiado es $P$. macrophylla HBK. (Rzedowski y Rzedowski, 1985). Zamudio (com. pers.) señala que se trata de un registro nuevo para el estado.

\section{ORCHIDACEAE}

Cranichis schaffneri Reichb.f. Chihuahua, Mpio. Ocampo, PNCB, in canyon along Río Basaseachic, in shaded woods. 4 Oct 1987, Spellenberg, Soreng, Corral \& Lebgue 8815 (NMC, TEX); 12 Sep 1987, Spellenberg, Jewell \& Columbus 9331 (NMC, MEXU). McVaugh (1985) indica que la especie se distribuye desde Sinaloa y Durango hasta Guatemala. Estas colectas al parecer representan el primer registro para Chihuahua.

Goodyera striata Reichb.f. Chihuahua, Mpio. Ocampo, PNCB, Divisadero overview to Rancho San Lorenzo Hotel, 26-27 Aug 1986, Martin, Moore \& Titley 86-100 (ARIZ, NMC); Todsen B20, 1/2 mi from campground, 2 Aug 1988 (NMC). La especie crece en bosque de pino y encino. McVaugh (1985) indica que esta orquídea se conoce desde Centroamérica hasta Hidalgo y Jalisco. Las colectas de Basaseachic extienden la distribución conocida hasta alrededor de $900 \mathrm{~km}$ hacia el norte.

Malaxis novogaliciana González Tamayo ex McVaugh. Chihuahua, Mpio. Ocampo, PNCB, on steep N-facing gully at overlook, 3 Oct 1986, Spellenberg, Soreng, Corral \& Lebgue 8680A (NMC, MEXU); along trail to falls, under oaks, 1 Aug 1988, Todsen B13 (NMC); 2 Aug 1988, Todsen B17 (NMC). Es común encontrar junto a esta especie a Malaxis corymbosa (S. Wats.) Kuntze. McVaugh (1985) indica que M. novogaliciana se conoce solamente del norte de Nayarit, del sur de Zacatecas, de Aguascalientes y de Jalisco. Los registros de Chihuahua se localizan aproximadamente a $650 \mathrm{~km}$ al norte del área previamente conocida.

\section{POACEAE}

Blepharidachne bigelovii (S. Wats.) Hack. Chihuahua, Mpio. Juarez, ca. $5 \mathrm{~km} \mathrm{SW}$ of Cd. Juarez in the extreme $\mathrm{N}$ end of Sierra Juarez, elev. ca. $1250 \mathrm{~m}, 17 \mathrm{Apr} 1992$, Spellenberg and Bacon 10992 (CIIDIR, MEXU, NMC, NY). Correll and Johnston (1970) 
indican que esta especie existe en "Trans-Pecos" y en Coahuila, pero no la citan de Chihuahua. Lebgue y Valerio (1991) no incluyen esta especie. Aparentemente, este es el primer registro para Chihuahua.

Eragrostis pringlei Mattei in Mattei \& Tropea. Chihuahua, Mpio. Guerrero, $11 \mathrm{~km} \mathrm{E}$ of Tomochic on arid flat, 6 Oct 1986, Spellenberg, Soreng, Corral \& Lebgue $8889(\mathrm{CHIH}$, ESAHE, ID, MEXU, NMC, NY). De acuerdo con McVaugh (1983), esta planta anual se localiza en Zacatecas, Jalisco y Michoacán y no se incluye en el trabajo de Lebgue y Valerio (1986), pero si el de Lebgue y Valerio (1991) basándose en este registro. La especie se encuentra también en PNCB (in clay mud around edge of now nearly dry temporary pool on flat, near hotel, 25 Oct 1992, Spellenberg 11674 (ARIZ, MEXU, NMC, NY). Las nuevas localidades representan una extensión en el área conocida de la especie en alrededor de $700 \mathrm{~km}$ al norte.

Muhlenbergia flaviseta Scribn. Chihuahua, Mpio. Ocampo, PNCB, on nearly barren rock at overlook, elev. ca. 2100 m, 3 Oct 1986, Spellenberg, Soreng, Corral, Lebgue 8666 (ARIZ, CHIH, ESAHE, NMC). La especie no se menciona para Chihuahua en el trabajo de Lebgue y Valerio (1986), pero se ha incluido en la revisión (Lebgue y Valerio, 1991), basándose en este registro. La colecta amplía la distribución geográfica conocida en aproximadamente $600 \mathrm{~km}$ al norte, dado que el taxon se había citado únicamente para la Sierra Madre de Durango. Otros ejemplares de la misma localidad (en PNCB) son los de Nesom y Vorobik 5643c, 5699 (TEX).

\section{ROSACEAE}

Amelanchier utahensis Koehne. Chihuahua, Mpio. Ocampo, PNCB, in canyon along Río Basaseachic, on shaded base of NW-facing steep slopes, 25 Apr 1986, Spellenberg, Soreng, Corral \& Lebgue 8435 (NMC). Kearney y Peebles (1960) establecen que la especie llega, hacia el sur, hasta la región este-centro de Arizona, mientras que Correll y Johnston (1970) indican que se extiende hasta el oeste de Texas y el norte de Baja California, tomando en cuenta lo que señala Jones (1964). Nuestra colecta aparentemente representa el primer registro para Chihuahua, el cual se localiza aproximadamente $400 \mathrm{~km}$ al sur de la latitud del oeste de Texas y del norte de Baja California.

Rubus palmeri Rydb. Chihuahua, Mpio. Ocampo, PNCB, along trail down SW-facing slope to base of falls, 26 Apr 1986, Spellenberg, Soreng, Corral \& Lebgue 8459 (ESAHE, MEXU, NMC). Especie previamente citada para Durango, Sinaloa, Nayarit y Jalisco (Standley, 1922). Los especímenes de esta colecta se identifican fácilmente en los trabajos de Standley (1922) y Rydberg (1913, p. 456), aunque difieren en la presencia de algunos tricomas en las drupillas y no son glabras, como lo menciona Rydberg.

\section{SCROPHULARIACEAE}

Penstemon havardii A. Gray. Chihuahua, Mpio. Praxedis G. Guerrero, Sierra San Ignacio, canyon $6.4 \mathrm{~km}$ SSW of Esperanza, 31'16' N, 10600' W, 1200-1400 m elev., 10 Apr 1983, R. D. Worthington and R. Corral-Díaz 9724 (NMC; UTEP); Mpio. Cd. Juarez, Sierra Juarez on W side of Cd. Juarez, ca. $6 \mathrm{~km}$ S of US-Mexico border, 106 $\mathrm{W}, 31^{\circ} 42^{\prime} \mathrm{N}$, elev. ca. 1380 m, N-facing limestone cliffs, 27 Dec 1991, Spellenberg and Bacon 10978 (MEXU, NMC); 17 Apr 1992, 10993, (CIIDIR, MEXU, NMC, NY - esta colecta fue referida con el 
Spellenberg, et al.: Nuevos Registros de Plantas de las Montañas del Norte de México

número erróneo '10093'). Correll y Johnston (1970) indican que la especie es endémica de la parte oeste de Texas. Aparentemente, este es el primer registro para México.

\section{AGRADECIMIENTOS}

Los autores agradecen a los siguientes individuos por su colaboración en la identificación de las plantas de algunas familias: Apiaceae - Dr. Lincoln Constance (UC); Eriocaulaceae - Dr. Antonio Lot (MEXU); Lentibulariaceae - Biól. S. Zamudio (IEB); Orchidaceae - Dr. G. A. Salazar (AMO) y Dr. T. K. Todsen (NMC); Poaceae - Dr. John Reeder (ARIZ).

\section{LITERATURA CITADA}

Barneby, R. C. 1964. Atlas of North American Astragalus. Mem. N. Y. Bot. Gard. 13: 1-1188.

Bayer, R. J. y G. L. Stebbins. 1987. Chromosome number, patterns of distribution, and apomixis in Antennaria (Asteraceae: Inuleae). Systematic Botany 12: 305-319.

Correll, D. S. y M. C. Johnston. 1970. Manual of the vascular plants of Texas. Texas Research Foundation; Renner. 1881 pp.

Gentry, H. S. 1942. Rio Mayo plants. Carnegie Institute of Washington, publication 527. Washington, D.C. $328 \mathrm{pp}$.

González Elizondo, M., S. González Elizondo y Y. Herrera Arrieta. 1991. Listados florísticos de México IX. Flora de Durango. Instituto de Biología, Universidad Nacional Autónoma de México. México, D.F. $167 \mathrm{pp}$.

González Villarreal, L. M. 1986. Contribuciones al conocimiento del género Quercus en el estado de Jalisco. Colección Flora de Jalisco. Instituto de Botánica, Universidad de Guadalajara. Zapopan. $240 \mathrm{pp}$.

Hickman, J. (ed.). 1993. The Jepson manual - higher plants of California. University of California Press. Berkeley. $1400 \mathrm{pp}$.

Jones, G. N. 1946. American species of Amelanchier. University of Illinois (Urbana). Biol. Monogr. 20: 1-126.

Kearney, T. H. y R. H. Peebles. 1960. Arizona flora (2nd. ed. and supplement by Howell J. T. y E. McClintock). University of California Press, Berkeley. 1085 pp.

Lebgue, T. y A. Valerio. 1986. Manual para identificar las gramíneas de Chihuahua. Talleres Gráficos del Gobierno del Estado de Chihuahua. Chihuahua, Chih. 231 pp.

Lebgue, T. y A. Valerio. 1991. Gramíneas de Chihuahua. Universidad Autónoma de Chihuahua, Chihuahua, Chih. $301 \mathrm{pp}$.

Lot, A., A. Novelo y P. Ramírez-García. 1986. Listados florísticos de México. V. Angiospermas acuáticas mexicanas 1. Universidad Nacional Autónoma de México, Instituto de Biología. México, D.F. $60 \mathrm{pp}$.

Mathias, M. E. y L. Constance. 1976. Umbelliferae. In: Harling G. y B. Sparre (eds.). Flora of Ecuador No. 145. Univ. Goteborg. Stockholm. pp. 1-71.

McVaugh, R. 1983. Flora Novo-galiciana, a descriptive account of the vascular plants of western Mexico. Gramineae. University of Michigan Press. Ann Arbor, Mich. Vol. 14. 436 pp.

Moldenke, H. N. 1937. Eriocaulaceae. North Amer. Flora 19: 17-50.

Moldenke, H. N. 1975. Additional notes on the Eriocaulaceae. LVII. Phytologia 32: 458-470.

Nesom, G. L. 1989. The erigerons (Compositae: Astereae) of Basaseachic National Park, Chihuahua, Mexico, including a new species. Phytologia 66: 471-475. 
Rydberg, P. A. 1913. Rosaceae (pars). North Amer. Flora 22: 389-480.

Rzedowski, J. 1978. Vegetación de México. Editorial Limusa, México, D.F. 432 pp.

Rzedowski, J. y G. C. de Rzedowski. 1979. Flora fanerogámica del Valle de México. Vol. 1. Compañía Editorial Continental, S.A. México, D.F. 403 pp.

Rzedowski, J. y G. C. de Rzedowski. 1985. Flora fanerogámica del Valle de México. Vol. 2. Publ. 15. Instituto de Ecología, A. C. México, D.F. 674 pp.

Standley, P. C. 1922. Trees and shrubs of Mexico. Contr. U.S. Nat. Herb. 23(2): 171-515.

Woodson, R. E. 1954. The North American species of Asclepias L. Ann. Missouri Bot. Gard. 41: 1211. 